\title{
INCLUSIÓN: PRINCIPIO DE CALIDAD EDUCATIVA DESDE LA PERSPECTIVA DEL DESARROLLO HUMANO
}

\author{
William Delgado Montoya \\ Docente en la Escuela de Administración Educativa de la \\ Universidad de Costa Rica \\ San José, Costa Rica
}

Recibido 29-III-2007 • Aceptado 13-XI-2007 • Corregido 11-XII-2007

"El cambio planificado implica una decisión consciente y positiva para producir una diferencia deseada".

Hanna (citado por Mora, 2005).

\begin{abstract}
Resumen: Este ensayo propone un acercamiento al concepto de inclusión como calidad de la educación desde la perspectiva del desarrollo humano. En este sentido, plantea una discusión sobre la participación del estado social, el derecho de la ciudadanía y los factores económicos hegemónicos en el marco de la globalización, que favorecen los mecanismos de exclusión: pobreza, acciones políticas elitistas, discriminación étnica, segregación cultural, participación de organismos internacionales en la toma de decisiones político-económicas nacionales y otros, limitando la gestión de un Estado de corte benefactor dentro de una estructura social. Además, considera el centro escolar como un espacio de interacciones entre los individuos, que promueve una humanización del sistema educativo para y por las personas. Asimismo, se busca inquietar al lector al esbozar las diferentes posiciones teóricas del pensamiento de la exclusión versus inclusión, aclarando la diferencia sustancial del compromiso de la gestión educativa en el contexto nacional, reflejado en diferentes estratos tanto económicos, políticos como sociales y culturales. Resalta el propósito de un enfoque inclusivo en la educación como parte de la aspiración en la administración de la educación por la calidad. Se justifican entonces los centros educativos incluyentes, donde el contexto de aprendizaje de los estudiantes y las estudiantes atiende las diferencias individuales de manera que sean consideradas dentro del desarrollo integral curricular, retomando las interacciones humanas que
\end{abstract}

En la actualidad, la sociedad en general y, principalmente, la costarricense, se enfrenta a la vivencia de transformaciones sociales y económicas, legitimadas tanto en el sector político como en el educativo; pues es un hecho que la educación al servicio de los sectores dominantes, promueve la subordinación de las otras clases sociales y que el poder estatal no sea reconocido socialmente por la colectividad, lo que conlleva a la ausencia de autoridad, porque pierde legitimidad; entonces, el Estado disminuye su capacidad de acción, por lo tanto, su poder es débil y carece de dominio económico.

En este sentido, Bazdresch (citado por Cordero, 2004, p. 35) cita que:

La relación entre la educación y la pobreza es una relación construida. No es obvia o "natural", no obstante, la idea muy difundida acerca de que la educación es una de las formas privilegiadas de evitar y/o salir de la pobreza. Las ideas de la pobreza y educación son constructos a supuestos e intenciones 
constantemente se modifican, para buscar estrategias $y$ proyectos más efectivos y eficaces en el proceso administrativo, que faciliten una acción propositiva ante el cambio en la educación. Así, el alcance de soluciones a los problemas se logra de una manera altruista, holística; eso implica un rol dinámico, democrático y multifacético que involucra al lector y el compromiso del administrador en las organizaciones educativas para lograr una gestión asertiva.

Palabras clave: Educación, sociedad educadora, segregación, hegemonía, inclusión, exclusión, gestión educativa, globalización, currículo, liderazgo, sinergia, aprendizaje, desarrollo humano, cultura y organización educativa.

\begin{abstract}
This paper proposes an approach to the concept of inclusion as the characteristic of quality on education from the perspective of human development. In this sense, it states a discussion related to the involvement of the social state, citizenship's rights and economic hegemony in the context of globalization that supports the mechanisms of exclusion: poverty, elitist policy actions, ethnic discrimination, cultural segregation, participation of international organizations in the decision-making process of economic politics of the national context and others. Thus, these mechanisms restrict the operation of a State within a social structure. Also, it considers the school as an area of interaction that promotes a humanistic system for education in favor of all the people. Moreover, it tries to create curiosity on the reader to outline the different theoretical positions of inclusion against exclusion, and, clarifying in this way the great difference of the educational management commitment in the national context reflected in different economic, politic, cultural, and social strata. It emphasizes the purpose of an inclusive approach in education as part of the desire in the administration of education in order to get the best quality. So, it justifies the educational inclusive centers, where the context of the students' learning considers individual differences within the holistic development curriculum, taking into account the human interactions that are constantly changing, to seek more effective strategies and projects in the administrative process that provide actions to carry out the changes in education.

As a result, the scope of solutions to the problems is accomplished in an altruistic and holistic way that implies a dynamic, democratic, and multi-faceted role that involves the reader and the administrator's commitment for educational organizations to achieve an assertive management.
\end{abstract}

Key words: Education, a teaching society, segregation, hegemony, inclusion, exclusion, educational management, globalization, curriculum, leadership, synergy, learning, human development, culture and educational organization. sociales que responden a los intereses de los diversos grupos sociales específicos.

Es decir, se puede comprender el papel "esperanzador" que juega la educación en las sociedades "pobres" o en vías de desarrollo como una forma para mejorar la calidad de vida; un poder adquisitivo de ciertos valores materiales y de estatus que, para la sociedad, resulta preponderante.

El contexto de la educación nacional refleja un proceso excluyente para "los pobres", ejemplo de ello es lo señalado en el Informe del Estado de la Educación Costarricense (Programa Estado de la Nación en Desarrollo Humano Sostenible, 2005), que menciona aspectos como la matrícula, el acceso a materiales o los gastos que resultan inaccesibles para las familias, porque no se ajustan a su ingreso salarial. Por esto, es importante citar el término "exclusión" social, para entender mejor la problemática que existe en el país y en América Latina en relación con este tema.

La exclusión social es un concepto que no hace mucho ha emergido como una herramienta analítica para las sociedades latinoamericanas, por lo tanto, según Sojo (citado por Macal, 2003), la literatura sobre tal término no es muy amplia. No obstante, junto con el fortalecimiento democrático en la región centroamericana a principios de los años noventa, el concepto empezó a ser empleado como un medio para examinar el fenómeno de la cohesión social como un mecanismo crítico del proceso democrático.

En la necesidad de profundizar en la interpretación de las dinámicas sociales, dados los niveles de desigualdad en las sociedades, principalmente en las latinoamericanas, el estado social no puede existir si no valora una articulación intercultural y respeto a la diversidad social.

El estado social se nutre de la desigualdad material de la sociedad capitalista y de la desigualdad organizativa de los actores sociales heterogéneos. Por medio de ese estado, “(...) se materializa el equilibrio 
de las fuerzas políticas y se configura una sociedad política ambivalente, que conserva, por un lado, la desigualdad social y económica con la igualdad jurídica y, por otro, crea las condiciones para que los excluidos cuenten con la garantía estatal para su reproducción social”, argumentó Huaylupo (2005, p. 138).

En Costa Rica, la pobreza ha provocado que deserten del sistema educativo muchos de los estudiantes y las estudiantes, a pesar de la legislación existente que garantiza la educación como un derecho humano fundamental. Miles de niños y jóvenes dejan las aulas para trabajar y aportar dinero a sus familias, por lo que truncan sus metas, limitan sus oportunidades de superación cultural, académica y económica y generan, así, el círculo denominado "de pobreza continua o círculo de pobreza".

En el Programa del Estado de la Nación (2007), en su Décimo Tercer Informe se afirma que el indicador de pobreza disminuyó en un punto porcentual con respecto al año anterior; sin embargo, esta variación no es significativa en términos sobre el ingreso real de los hogares con menos recursos y los escasos cambios en el perfil de los hogares pobres y vulnerables que están en la base de la pirámide social, no inciden sobre la permanencia del estudiante en las aulas de la educación formal.

En el contexto regional, se considera a la exclusión social de una manera más amplia, por ejemplo, Cordero en relación con este tema cita lo siguiente:

(...) la imposibilidad de una persona o de un grupo social para participar activamente en las esferas económicas, culturales, políticas o institucionales de la sociedad. Una escasez crónica de oportunidades y de acceso a servicios básicos de calidad, a los mercados laborales y de crédito, a condiciones físicas y de infraestructura adecuada, y al sistema de justicia (Cordero, 2004, p. 7).

La autora valora algunos de los factores de exclusión en América Latina, señalando la pobreza, la discriminación étnica, la economía como dependientes del régimen de órganos internacionales. Es decir, como bien se señala dentro de las oportunidades de apertura del capital humano, -talento humano actualmente-, los pobres no tienen las redes sociales ni los recursos culturales para superar su pobreza lo que trae consigo la exclusión social, laboral y la desesperación manifestada en posibles comportamientos destructivos en la juventud urbana que participan en agrupaciones como las "Maras o pandillas".

Igualmente, otro factor por retomar es la discriminación étnica, aspecto que puede contribuir a la exclusión social y es el tinte actual de miles de libros, publicaciones, simposios, mesas redondas, título de canciones y películas.

El panorama social incita a reafirmar que las desigualdades raciales y étnicas están ligadas a la pobreza, a los bajos indicadores de salud y educación, a las pocas oportunidades de generación de ingresos, al agotamiento de los recursos naturales, a la migración forzada y a la falta de acceso al conocimiento y a la información sobre sus derechos.

En el ámbito político, las deficiencias de la democracia traen como resultado la ausencia de participación en la vida política de grandes sectores sociales, ya que el "clientelismo" y la corrupción, junto con la baja capacidad organizativa de muchos grupos que representan a los pobres, limitan el diálogo y la participación pública (Huaylupo, 2005).

Finalmente, la fragmentación urbana y rural, así como la segregación residencial dentro de las ciudades socava el sentido de pertenencia social.

Actualmente, “(...) los procesos que gestaron el estado social en Costa Rica han perdido la fuerza y vitalidad democrática de su nacimiento. Las clases subalternas, sin capacidad organizativa, no tienen posibilidad de negociación ni de confrontación ante las fuerzas orgánicas del capital y del Estado. La división, o la atomización, de intereses en un atmósfera social individualista posibilita la realización de proyectos 
políticos, económicos y sociales ajenos a la colectividad nacional, sin oposición activa y significativa", argumentó Huaylupo (2005, p. 151).

Con el proceso de globalización la sociedad costarricense ha perdido su capacidad de dirigir y tomar decisiones propias, actualmente los organismos internacionales dan las pautas por seguir, sus miembros son sujetos para ser considerados candidatos a un préstamo.

$\mathrm{Al}$ ser Costa Rica una economía dependiente, lo único que le queda es seguir las condiciones impuestas por los organismos internacionales. Ya no es necesario el colonialismo como ocurrió en el siglo pasado, ahora la versión es el neocolonialismo, donde conquistar un país y someterlo, política, económica y culturalmente depende de los préstamos que permiten ejercer un dominio total desde la esfera comercial, económica, sociopolítica y promueven una imposición cultural.

A lo anterior se suma la globalización que ha impactado la sociedad en todos sus niveles, dándose relaciones no igualitarias, mucho menos podría decirse equitativas; así de acuerdo con Huaylupo (2005, p. 152) en relación con este fenómeno planetario, se ha llegado a la conversión de un Estado social en un "Estado Privado" de

(....) una transformación reaccionaria, es una vuelta caricaturesca al pasado, así como la renuncia expresa y manifiesta a la relativa autonomía clasista, para subsumirse a la voluntad de los propietarios del capital mundializado. Las aspiraciones nacionales, el desarrollo, el bienestar en esencia pluriclasista e interdependiente, ha sido reducida a los logros empresariales. Las más altas jerarquías del poder estatal han identificado el enriquecimiento empresarial con bienestar social o han subordinado el desarrollo y el bienestar social al crecimiento de las ganancias de empresarios mundiales.

El proceso de globalización, liderado por las corporaciones transnacionales $\mathrm{y}$ los organismos internacionales impulsan políticas económicas hegemónicas, diseñadas desde afuera, que operan en América Latina e implican reformas en los sistemas educativos, los cuales son los que reproducen el sistema y lo hacen global, al preparar a los trabajadores que necesitan esas grandes empresas, que representan "una mano de obra barata calificada" para los fines de esas transnacionales.

Por eso, en Costa Rica, se han variado los programas educativos, incorporando otras asignaturas (tecnologías) y se han formado colegios de diferentes modalidades como los colegios técnicos, científicos, ecoturísticos..., en los cuales se trata de dar herramientas a los jóvenes para que cuando salgan de la educación general básica, provean mano de obra técnica barata.

Es decir, el reto no es solamente hacer lo que se puede, no es solo la oferta educativa preconcebida, sino dar y hacer imperiosamente lo que hace falta y lo que se necesita, como acciones de inclusión en un sistema exclusivo (Miranda, Pérez y Smutt, 1999).

Por otro lado, a nivel político, la globalización transforma los estados con la adopción de una serie de políticas de carácter económico cuyo propósito es la liberación del mercado, de ahí la propuesta de acciones como tratados de libre comercio, reestructuración del Estado como agente económico que busca la privatización de empresas públicas, la inversión en materia social y la promoción de la libre movilidad del capital entre los países, eliminando todo tipo de proteccionismo estatal al ciudadano.

Evidentemente, esto al mismo tiempo, se sucede también en la esfera cultural con la pérdida de la idiosincrasia y de la identidad cultural de los pueblos por medio del fenómeno de la transculturalización; este hecho se manifiesta mediante la producción industrial de la cultura, su conocimiento tecnológico y el consumo diferido y segmentado de los bienes (Huaylupo, 2005; Macal, 2003).

Así al "indoculturalizar" el planeta de acuerdo con los valores, actitudes y creencia foráneas, se afectan en general las generaciones jóvenes de los países que sufren este fenómeno. 
Todos estos factores dictan una visión general de los factores económicos, institucionales, políticos y culturales que pueden empeorar y ocasionar los procesos incipientes de exclusión (CEPAL, citado por Macal, 2003).

Como producto de la exclusión social, la ciudadanía presenta “(...) la carencia de condiciones materiales básicas y de códigos para la realización de derechos junto con un mal sentido de cooperación y reciprocidad como la base del bien social común" (Macal, 2003).

En este sentido, la ciudadanía se basa en los derechos civiles, políticos y sociales y apunta a la relación entre el individuo y el Estado, entre el individuo y la sociedad. Es decir, los ciudadanos son tanto súbditos como actores que luchan por superar las limitaciones materiales básicas de la pobreza y encaminarse hacia la emancipación.

El concepto de "ciudadanía" se ha desarrollado a través de los siglos; desde la libertad para la participación política hasta el interés contemporáneo con los derechos de los ciudadanos. Estos cambios han mostrado una continua inclinación por la equidad, por una mayor inclusión social con la meta de cerrar la brecha de ingresos entre los ricos y los pobres, así como marcar la responsabilidad pública por los derechos sociales.

Sin embargo, Cordero (2004) comenta que, los argumentos del modelo de liberalización económica actual y las políticas que se encuentran en el núcleo de la mayoría de las economías latinoamericanas, promueven muy poco una mayor participación social, económica y política de la mayoría de latinoamericanos.

La autora citó que esta situación ya ha conducido a una creciente desilusión hacia el sector privado y a un creciente énfasis de la importancia del carácter público de la educación, del cuidado de la salud y de la provisión de servicios básicos.

Además, a fin de evitar la "fragmentación” de la ciudadanía, con base en la capacidad de acceso a los bienes y servicios públicos, se torna crítica la necesidad de contexto, la emergencia de "actores sociales", poseedores de derechos y que trabajen para la construcción de la ciudadanía, para lo que es fundamental el fortalecimiento de la inclusión.

\section{Vulnerabilidad social consecuencias en las transformaciones económicas asumidas por el Estado}

Estudios han mostrado que en la región centroamericana es muy alto el costo social de las políticas económicas actuales, que se refleja en el creciente nivel de desigualdades de ingresos durante las últimas dos décadas.

La pobreza se está volviendo cada vez más heterogénea debido al empobrecimiento de los sectores medios y bajos, lo que origina una gran zona de vulnerabilidad en los niveles de pobreza, emigración, economías de subsistencia, remesas, entre otros.

En términos de la creación de empleo es insuficiente, lo que resulta en un incremento en los puestos de baja productividad en el sector informal y altas tasas de desempleo.

De igual manera, en otras regiones del mundo como en América Latina la integración al mercado del trabajo y la redistribución de ingresos son dos determinantes importantes de inclusión o exclusión social.

En este momento, muchos países latinoamericanos se encuentran pasando por un proceso de profundización de desigualdades sociales que inevitablemente frustrarán los esfuerzos hacia la integración social en términos, por ejemplo, del ejercicio de derechos y ciudadanía.

Cordero (2004) señaló que el resultado de esto, dentro de las estructuras sociales de las sociedades latinoamericanas se torna más complejo, un grupo pequeño incluido versus un creciente sector vulnerable y un grupo muy numeroso excluido social y económicamente. 


\section{Segregación cultural: efecto de la indoculturalidad}

Sojo (citado por Macal, 2003) considera que la exclusión cultural debe ser comprendida como

El acceso diferenciado de sectores sociales a los beneficios del bienestar social y material cuando las causas no son estructurales. Las causas de la estratificación cultural se pueden dividir en dos categorías principales: especificidad subjetiva y acceso a bienes simbólicos. La primera incluye factores tales como género, antecedentes étnicos o de raza, edad y pertenencia a un grupo minoritario (homosexuales, incapacitados, inmigrantes y otros). En la segunda categoría, los bienes simbólicos se definen como los recursos e infraestructura que difunden información, conocimientos y valores y la capacidad del individuo para entenderlos.

Muchas de las sociedades latinoamericanas y del Caribe tienen una creciente brecha entre la integración simbólica y la material. Dada la naturaleza de las comunicaciones globales, muchos latinoamericanos tienen un mayor acceso a los bienes simbólicos, es decir, a las imágenes, los símbolos y los mensajes colectivos, mientras que, al mismo tiempo, ocurre una concentración de la riqueza y un acceso limitado a los beneficios económicos de la integración al mercado global.

Por lo tanto, las expectativas creadas por el proceso de modernización en términos de la integración material no se están realizando, en vez de ello, la globalización de la información y las comunicaciones han creado la ilusión de la integración simbólica que es socavada por la carencia de integración social.

En este sentido, el acceso diferenciado a la educación de calidad profundiza la brecha entre los sectores incluidos y los excluidos en términos de oportunidades de integración material y simbólica.

Las expectativas frustradas y la ausencia de mecanismos de movilidad social, especialmente entre la juventud urbana, a menudo resulta en niveles crecientes de violencia como un síntoma de desintegración social (Hopenhayn 1999; CEPAL 2000, citados por Macal, 2003).

\section{Exclusión social en el contexto latinoamericano de grupos étnicos}

No puede obviarse la situación en el contexto del fenómeno de la exclusión cultural, la relación entre la exclusión, la raza y el grupo étnico surgen como temas críticos en el entendimiento de la exclusión social y económica.

Macal (2003) menciona que algunas perspectivas útiles de 'Economía Sigma' en América Latina, basado en dos supuestos básicos. Primero, las personas que participan en los intercambios en el mercado no sólo tienen diferentes cantidades de bienes económicos sino que también tienen diferentes cantidades de bienes políticos y culturales.

Segundo, existe una jerarquía de mercados, en la cual los de trabajo, de crédito y de seguros (llamados "mercados básicos") juegan un papel crítico en la generación y reproducción de desigualdades.

Figueroa (citado por Cordero, 2004, p. 29) afirma que la "Teoría Sigma" analiza los factores de desigualdades en el largo plazo en una sociedad heterogénea. Según esta teoría, la historia es crítica lo que resulta en procesos económicos y sociales que afectan la tenencia actual de bienes (variables endógenas) que tenderán a reproducir las desigualdades sociales.

La evidencia empírica muestra que los países latinoamericanos con grandes poblaciones indígenas funcionan como la "Economía Sigma", en la que los grupos indígenas representarían el estrato social con la menor cantidad de bienes económicos, políticos y culturales, en relación con el grupo cultural predominante.

Los países de América Latina con grandes grupos étnicos son los más desiguales. Entre estos países, las áreas predominantemente indígenas se caracterizan por una "economía campesina" (sistema de 
mercados mal desarrollado y pocas innovaciones tecnológicas) (Cordero, 2004).

Esta situación se puede explicar mediante el uso del concepto de la exclusión social, comprendido como la carencia de oferta de mano de obra indígena en los mercados urbanos (exclusión económica), carencia de preocupación o interés por parte del gobierno para la provisión de bienes e infraestructura públicos en áreas indígenas (exclusión política) y educación de mala calidad (exclusión política y cultural).

Por lo tanto, la jerarquía de diversidad étnica y cultural existente, funciona como un mecanismo de exclusión y refuerza la incapacidad para acumular el capital social y económico, lo que reproduce futuras generaciones de exclusión, al perderse de vista el bienestar social (seres humanos como beneficiarios) como parte de un valor integrador del trabajo y principio del desarrollo humano sostenible.

Delors (1996) escribió que el desarrollo del bienestar humano tiene como finalidad una mejor calidad de vida en salud, medio ambiente, alimentación, agua potable, educación y nutrición.

La equidad y la igualdad entre los diferentes grupos sociales y sexos, dentro de la "aldea planetaria", puede lograrse mediante una sostenibilidad complementaria al desarrollo (proceso de calidad de vida, que se puede divisar en la disponibilidad y acceso a los recursos, salud, longevidad, auto respeto) como en la proporción de bienes y servicios materiales analizando el crecimiento económico, el comercio, los valores culturales de países en desarrollo e industrializados que reúnen la producción y la distribución, ampliación y aprovechamiento de la capacidad humana.

En este sentido se plantea, que la educación debe lograr que la humanidad pueda dirigir cabalmente su propio desarrollo, es decir, que cada persona se responsabilice de su destino con el fin de contribuir al progreso de la sociedad en la que vive.
Por ello, es oportuno citar el siguiente comentario de González (2000, p. 75) referente a la gestión educativa para la sociedad costarricense.

Forzar al personal (...) a cooperar, los vuelve más escépticos y se equivoca el camino hacia la coordinación de esfuerzos institucionales y educativos. Por lo tanto, la supervisión curricular y las intervenciones han de verse como un último recurso, y no como una prioridad esencial. Las habilidades para generar sinergia requieren de una colaboración significativa, mediante los "puentes de comprensión y una intervención efectiva.

\section{Humanización escolar: principio de una calidad de educación}

Ante la realidad descrita y que caracteriza el paisaje latinoamericano, surge la necesidad de un enfoque educativo basado en la valoración de la diversidad como elemento enriquecedor del proceso de enseñanza y aprendizaje, como consecuencia favorecedora del desarrollo humano.

El concepto de educación inclusiva es más amplio que el de integración y parte de un supuesto distinto por el que se relaciona con la naturaleza misma de la educación regular y de la escuela común (Cordero, 2004).

La educación inclusiva implica que todos los niños y las niñas de una determinada comunidad aprendan juntos independientemente de sus condiciones personales, sociales o culturales, incluidos aquellos que presentan alguna condición especial que les dificulta el aprendizaje.

Es decir, se trata de una escuela que no pone requisitos de entrada ni mecanismos de selección o discriminación de ningún tipo, para hacer realmente efectivos los derechos a la educación, a la igualdad de oportunidades y a la participación como parte fundamental del derecho a recibir una educación.

La UNESCO propone el concepto de educación inclusiva, definida por Arnaiz (citado por Delors, 1996, p. 9) como 
Primero, es una actitud, un sistema de valores y creencias, no una acción ni un conjunto de acciones. Una vez adoptada por una escuela o por un distrito escolar, debería condicionar las decisiones y acciones de aquellos que la han adoptado. La palabra incluir significa ser parte de algo, formar parte del todo. Excluir, el antónimo de incluir, significa mantener fuera, apartar, expulsar. Estas definiciones empiezan a servir de marco para el creciente movimiento de construcción de las escuelas inclusivas. El mismo significado de los términos inclusión y exclusión nos ayuda a entender la educación inclusiva.

En una educación inclusiva, los valores importantes subyacentes son los de aceptación, pertenencia y comunidad, las relaciones personales, la interdependencia además de la independencia, y la consideración de los profesores y de los padres como una comunidad de aprendizaje.

Una escuela inclusiva, como principio de un aula humanizada, un rostro educativo de bienestar para la colectividad, ve a todos los alumnos como capaces de aprender, animando y buscando honrar todos los tipos de diversidad, como una oportunidad para aprender sobre lo que nos hace humanos, incluyendo las diferencias, inteligencias múltiples y un aprendizaje para la vida.

La inclusión se centra, pues, en cómo apoyar las cualidades y las necesidades de cada uno y de todos los estudiantes en la comunidad escolar, para que se sientan bienvenidos y seguros y alcancen el éxito.

Stainback, S. y Stainback, W. (1992), definieron una escuela inclusiva como aquella que educa a todos los estudiantes dentro de un único sistema educativo, proporcionándoles programas educativos apropiados, estimulantes y adecuados a sus capacidades y necesidades, además de brindarles cualquier apoyo y ayuda que tanto ellos como sus profesores puedan necesitar para tener éxito.

Sin embargo, una escuela inclusiva va más allá de todo esto, ya que es un lugar al que todos pertenecen, donde todos son aceptados y son apoyados por sus compañeros y por otros miembros de la comunidad escolar para satisfacer sus necesidades educativas.
Uno de los rasgos esenciales de una escuela inclusiva es el sentido cohesivo de comunidad, la aceptación de las diferencias y la respuesta a las necesidades individuales. Macal (2003) comenta algunos aspectos que rescata lo señalado por Sapon-Shevin, con respecto a una educación excluyente, destacando por ejemplo:

1. El mensaje "si eres distinto debes marcharte", puede hacer que el sentimiento de seguridad en el aula que tiene el niño o la niña se tambalee.

2. Excluir o sacar a los estudiantes que son identificados públicamente como diferentes hace más difícil promover la educación pluricultural y una respuesta positiva ante las diferencias.

3. Las comunidades cohesivas requieren una comunicación abierta sobre las diferencias. Si las diferencias no se discuten abiertamente, se puede crear un clima de desconfianza y de alienación.

4. El ir y venir de los niños puede interrumpir el discurrir de la clase y dificultar la tarea de los profesores para establecer un grupo cohesivo.

5. Excluir a los niños del aula normal para que puedan satisfacer sus necesidades especiales pone en entredicho el sentimiento de los profesores de verse a sí mismos como responsables o capaces de enseñar a un grupo diverso.

Es importante enfatizar que moverse hacia la inclusión y aceptarla supone un proceso, que implica cambios en la filosofía, el currículo, la estrategia de enseñanza y la organización estructural. Tales cambios no sólo afectan a los niños clasificados como "discapacitados", sino que van más allá del alcance de lo que tradicionalmente ha sido considerado como educación especial.

En Costa Rica, la inclusión es lo opuesto a la segregación y al aislamiento 
por la incorporación de la Ley 7600. Si se vuelve la vista atrás, se recordará cómo la educación especial creó un subsistema segregado, del que se ha extraído un fuerte mensaje de que los alumnos, debido a la alta especialización que requerían para ser atendidos, no "encajaban" en el sistema ordinario de la educación, para citar un ejemplo.

La creciente diversidad de estudiantes en el sistema educativo costarricense es un importante tema de debate y preocupación. Entre las diferencias se encuentran la cultura, la religión, el sexo, la discapacidad, la preferencia sexual, el estado socioeconómico, el marco geográfico y muchas más que denotan la multiculturalidad existente en la escuela.

Sin embargo, frente a esta realidad, a menudo, se encuentra que la diversidad es entendida como un problema más que como una maravillosa oportunidad de aprender sobre la variedad de vida de otras personas y también sobre lo que significa ser humano, el juicio de ser incluido, valorado y respetado por lo que se es, en un mundo diverso y plural por naturaleza.

\section{Asuntos por resolver: la equidad e inclusión}

La "sociedad educadora" como la llama Álvarez (2001, p. 13) dimensiona la educación como una unidad donde “(...) todos los sistemas de educación formal forman parte integral del sistema mundial de la educación, (...) una comunidad intelectual, si se pone al margen es como (...) un miembro humano (...) que hubiera dejado de circular la sangre (...)".

En este sentido, la multicultaridad es una realidad en nuestra sociedad debido a los movimientos migratorios de numerosos grupos sociales de características culturales específicas, que están determinando que diversas culturas compartan el mismo territorio, la misma sociedad y los mismos centros educativos.
Esa es una situación que no está siendo valorada de igual forma en los distintos contextos en que se produce y que muestra dos polos bien diferenciados de una misma situación. Así, mientras encontramos en el polo positivo la integración intercultural, que supone el respeto a la diversidad que implica pertenecer a una cultura minoritaria y el reconocimiento de los principios de igualdad de derechos y de deberes de los miembros de distintas culturas que conviven en una sociedad plural; por otra parte, en el polo opuesto se sitúa la asimilación, práctica bastante habitual que niega los rasgos propios de la cultura minoritaria para ser absorbida por la mayoritaria; la segregación, caracterizada por la exclusión o aislamiento social de los grupos minoritarios y el racismo, que implica la negación de toda forma de contacto o experiencia cultural entre grupos de distintas culturas.

Se puede decir que en estas últimas prácticas los componentes que definen el término cultura, como conocimientos, valores y comportamientos son conculcados, impidiendo que grupos sociales que conviven puedan compartir un mismo ambiente e ir resolviendo los problemas que puedan presentárseles.

Además, la diversidad social es otro elemento por ser discutido, debido a que la pertenencia a una clase social determinada tiene gran influencia para sus miembros en aspectos tales como el acceso a los estudios, la organización de los procesos de enseñanza y aprendizaje que se lleven a cabo según el centro educativo al que se asista y los resultados académicos que se obtengan.

De esta manera, el modelo del "déficit cultural" aparece ligado a la desventaja social y defiende que los alumnos en esta situación proceden de familias o de grupos sociales que viven en situación de discriminación económica o de marginación social; por tanto, desde su nacimiento estos niños están expuestos a normas, lenguaje, valores y expectativas que determinan situaciones de desventaja con respecto a sus iguales, así como falta de estimulación y experiencias (Cordero, 2004). 
Por otra parte, el modelo de las diferencias culturales destaca los rasgos culturales propios de los grupos étnicos minoritarios o de las clases sociales más bajas (normas, valores, sistemas de creencias y estilos de comportamiento) y trata de llamar la atención del escaso reconocimiento que se hace de ellos en los centros educativos.

Ese hecho se relaciona directamente con el mayor fracaso escolar que los grupos minoritarios o de clases sociales más bajas tienen frente a los grupos mayoritarios y que evidencia la descoordinación existente entre la cultura escolar y la cultura de estos colectivos, provocando un menor rendimiento en los alumnos de escasos recursos y un mayor riesgo de abandono escolar. El funcionamiento homogeneizador y asimilador del sistema educativo indudablemente también contribuye a acentuar esa situación.

Otro aspecto relevante es la diversidad de sexos que se ha convertido en un elemento de desigualdad y discriminación en muchos contextos y ocasiones. A lo largo de la historia y aún actualmente, no se ha conseguido la igualdad de oportunidades para las mujeres en el ámbito de la educación. Igualdad no sólo referida al acceso al trabajo o a la educación, sino también a un tratamiento equitativo a través del currículo en cuanto a objetivos, contenidos, estilos de aprendizaje, materiales y libros de texto, expectativas sobre los alumnos y forma de organización escolar.

La diversidad que se quiere considerar bajo el epígrafe, referido a las diferencias que se producen en el aprendizaje de los alumnos debido a la particularidad de las dimensiones cognitivas, motivacionales, afectivas y relacionales con la Ley 7600 en nuestro contexto nacional.

La diversidad cognitiva guarda relación con la variabilidad de los alumnos en cuanto a diversidad de procesos, estrategias, estilos de aprendizaje y de conocimientos básicos, que imprime una cierta variabilidad a las condiciones de aprendizaje de los alumnos.
La motivación hacia lo académico está relacionada con la consecución de metas, en tanto y en cuanto la actividad de la persona se oriente hacia la consecución de objetivos con éxito. Esta condición se liga a la valoración positiva de la propia competencia y a las capacidades metacognitivas o de autorregulación. La influencia de los factores emocionales en el aprendizaje tiene cada vez más credibilidad por cuanto los sentimientos y las capacidades afectivas están íntimamente implicados en el proceso de aprender.

La propia personalidad, el autoconcepto, la autoestima, las expectativas que el alumno tiene sobre el profesor, así como el concepto y las expectativas que el profesor tiene sobre el propio alumno juegan un papel fundamental y determinante en el proceso de enseñanza y aprendizaje.

El concepto de necesidades educativas especiales (n.e.e.) ha abierto una nueva visión sobre las personas con alguna deficiencia. Las n.e.e. asociadas a discapacidad se refieren a aquellos alumnos y alumnas que se enfrentan al proceso de enseñanzaaprendizaje con limitaciones sensoriales, cognitivas o dificultades de aprendizaje. Estas necesidades pueden verse aumentadas o disminuidas según el modelo de enseñanza que se lleve a cabo en los centros educativos, ya que es muy importante la consideración de sus experiencias previas, el tipo de currículo diseñado o su dificultad para incorporarse al ritmo de aprendizaje establecido.

Además, la desigualdad en el acceso al conocimiento se enfrenta con la reivindicación de los derechos humanos y la lucha contra la magnificación de las diferencias, que ha traído consigo la reivindicación de conceptos de equidad, justicia e igualdad como propiciadores de concreciones diversas en la situación de desigualdad educativa.

El término equidad enfatiza la diversidad de posibilidades de los alumnos y las alumnas a orientar las decisiones en el ámbito educativo de acuerdo con ellos y con ellas (Cordero, 2004). 
La justicia debe estar presente en la acción educativa con el objeto de responder a las aspiraciones de todos los ciudadanos y las ciudadanas y; la noción de igualdad considera aspectos como la necesidad de igualdad de oportunidades, igualdad de acceso en el planteamiento educativo y en los resultados.

Las desigualdades presentes en el sistema educativo son un tema complejo que necesita analizar el origen y las interacciones existentes entre las posibles desigualdades. Por ello, es necesario considerar las desigualdades iniciales procedentes de condiciones sociales y culturales distintas, los mecanismos que tienden a mantener esas desigualdades a lo largo del proceso de escolarización, así como comprender los factores más estrechamente relacionados con la calidad de la enseñanza, con el fin de obtener una mayor interpelación entre ambos conceptos.

Durante mucho tiempo, el origen social ligado a problemas económicos subyacentes ha sido considerado como la causa principal de las posibles desigualdades en el acceso a la educación y en el rendimiento académico. No obstante, desde los años sesenta y setenta, las nuevas consideraciones de la sociología de la educación han determinado que debe tenerse en cuenta, junto con el origen social, el papel de los centros educativos como reproductores de la estructura y los valores de la clase dominante.

Consecuentemente, las pocas oportunidades en el acceso a la educación de las clases sociales más desfavorecidas y de la mujer, la no presencia de esos sectores de la sociedad en los distintos niveles educativos, especialmente en la educación secundaria, los menores logros académicos y las escasas posibilidades de empleo en profesiones intermedias y superiores son factores evidentes de las desigualdades generadas por los propios centros educativos.

Estas desigualdades en el acceso a la educación y a lo largo del todo el proceso educativo han determinado la participación de alumnos con dificultades o procedentes de minorías étnicas en programas especiales menos valorados académica y socialmente, su participación en clases de refuerzo o especiales, influyen negativamente en la autoestima de estos alumnos y en la organización de la enseñanza en grupos homogéneos.

Otros factores que han contribuido a la desigualdad, además de la clase social y el centro educativo, son los aspectos familiares, culturales y lingüísticos. Muchas veces las diferencias proporcionadas por la falta de herencia cultural e información, el escaso contacto con la cultura dominante, la falta de aptitudes y dominio de la lengua contribuyen a reforzar las desigualdades sociales.

Según Macal (2003), los estudios de Bernstein en los años comprendidos entre 1971 y 1974 demostraron que existe una clara relación entre la clase social de procedencia de los alumnos, el uso del lenguaje y el proceso de socialización de la familia con los iguales, en la escuela y en el trabajo.

Por esta razón, los centros educativos, al estar organizados con valores, normas y códigos lingüísticos de la clase media plantean enormes barreras para aquellos alumnos de la clase trabajadora, con dificultades de aprendizaje o provenientes de minorías étnicas y culturales, contribuyendo a mantener las diferencias sociales.

Igualmente, la variable profesor ligada a su mayor o menor competencia para dar una respuesta educativa acorde a las características de sus alumnos ofrece datos relevantes a este respecto. Durante más de veinte años ha permanecido la creencia de que cualquier enseñanza es tan eficaz como cualquier otra y que los profesores con una formación y experiencia desigual son igualmente buenos para todos los alumnos.

El movimiento sobre escuelas eficaces está aportando nuevos planteamientos de gran interés para este tema que reclama atención sobre la diversidad. 


\section{Apreciaciones finales}

El papel de los centros educativos como espacios institucionales debe propiciar la capacidad de asegurar la igualdad de oportunidades a todas las personas, garantizando una formación y progreso social a partir de una gestión educativa que atiende la diversidad.

En dichos contextos educativos, por ejemplo, la educación intercultural no es, ni debe identificarse con la educación de niños y niñas pertenecientes a minorías étnicas, sino con la educación de todos los estudiantes y las estudiantes para convivir y colaborar dentro de una sociedad pluricultural (Muñoz, 1997, citado por Macal, 2003).

De igual manera, el currículo que requiere la educación intercultural organiza conceptos y contenidos alrededor de las contribuciones, perspectivas y experiencias, confronta temas sociales que incluyen la etnia, la clase socioeconómica, el sexo, la homofobia, la n.e.e. y la discapacidad (Grant y Tate, 1995, referidos por Macal 2003).

Otra de las finalidades de un currículo pluricultural es incluir las contribuciones de los guetos para construir una identidad etnorracial, especialmente en las áreas de ciencias sociales, fomentando la comprensión de diferentes grupos sociales, con el fin de mejorar las relaciones sociales en una sociedad cada vez más diversa y compleja.

Marchesi y Martín (citados por Cordero, 2004, p. 37) comprenden el currículo como lo que

(...) se plantea a todos los alumnos para que todos aprendan quiénes son los otros y qué debe incluir, en su conjunto y en cada uno de sus elementos, la sensibilidad hacia las diferencias que hay en la escuela. La educación en la diversidad tiene que estar presente en todo el currículo y en todo el ambiente escolar.

En resumen, ante una valoración social, cultural y económica de nuestro contexto nacional, regional y continental, se constata que vivimos una realidad donde la pobreza y las diferencias de sexo, grupo étnico, cultura o el acceso al conocimiento continúan siendo motivos de exclusión para las personas que no poseen los requisitos que, dentro de una determinada sociedad y en un momento histórico definido, son impuestos por quienes dirigen en los ámbitos mencionados.

La relación entre sociedad y currículo es estrecha; por eso, el currículo deberá institucionalizar los valores sociales, morales y culturales que practican los actores y las actrices de la sociedad, por lo tanto, una sociedad excluyente plasma sus opciones en una "Escuela Excluyente".

Los "Centros Educativos Incluyentes" pretenden un cambio: el atender la diversidad de las personas, fortaleciendo el talento humano y brindando espacios de acceso al conocimiento como parte de una de las formas más efectivas de ejercer el poder en las sociedades.

La superación de la pobreza continúa siendo el principal objetivo de las autoridades estatales y de las instituciones educativas, ya que es considerada como el principal motivo de exclusión social, sobre todo en nuestras sociedades latinas.

El desarrollo humano es un proceso conducente a la ampliación de las opciones que disponen las personas, por tanto, la educación incluyente deberá propiciar este principio, para propiciar una calidad de vida reflejada en los accesos a los diferentes niveles de educación, una salud prolongada, donde no se objete que la educación sea solamente un medio proveedor del mundo económico de personas calificadas, sino que favorezca el desarrollo de los talentos y aptitudes de cada individuo.

Por tanto, la administración de la educación centrada en la inclusión como principio del desarrollo humano permitirá a cada persona la posibilidad de adquirir una educación permanente, global e integradora, que cubra todos los aspectos de su vida diaria, la destreza profesional y los valores morales y ciudadanos, que debe concebirse como un proceso permanente.

Bien lo señaló Suárez, (1998): 
(...) una educación para todos que satisfaga para todos el reto de ponerse permanentemente al día”, debido a que las exigencias del líder en el actual siglo XXI lo convierten gestor o gestora que aprende a conciliar entre sus ideales y el de los que están a su alrededor, entendiendo que la organización educativa es un "ecosistema", una casa que siempre necesita cuidado, limpieza y orden, aunque implique tomar acciones que no sean agradables de asumir como parte de una acción ante el cambio. De la Torre y otros (1998, p. 6) citan que
Delors, J. (1996). La educación encierra un tesoro. Madrid: Editorial Santillana.

González, L. (2000). El efecto de la sinergia en la gestión administrativa. Revista Educación, 2(24), 71-80.

Huaylupo, J. (2005). Tiempo-espacio en la constitución estatal. Una perspectiva desde la sociedad costarricense. Revista de Estudios Latinoamericanos, 40, 129-168.

Macal, H. (2003, abril). Escuela incluyente. Ponencia presentada en el VI Encuentro CERCA y CIMACMesoamérica, (Memoria Digital [CD], sin paginación). San José, Costa Rica: Comunidad Salesiana.

Miranda, L., Pérez, A. y Smutt, M. (1999, Noviembre 22-25). El Salvador, Poligono Industrial Don Bosco: sistematización de experiencias. Documento presentado en el Seminario Regional organizado por IIPE, INA y UCR.

Arnaiz, P. (1995, abril 28). Integración, segregación e inclusión. Ponencia presentada en la XXII Reunión Científica de AEDES "10 años de integración en España: análisis de la realidad y perspectiva de futuro". España, MURCIA.

Bustela, M. (1998). Política social e igualdad, todos entran: propuesta para una sociedad incluyente. Bogotá: Santillana-UNICEF.

Cordero, H. (2004). Educación inclusiva. Revista EDUCARE, VI, 23-38.

De la Torre, S., Jiménez, B., Tejada, J., Carnicero, P., Borrell, N. y Medina, J. (1998). Cómo innovar en los centros educativos: estudios de casos. Madrid: Editorial Escuela Española.
Mora, M. (2005). Supervisión y evaluación de la gestión educativa. Mimeografiado. Universidad Estatal a Distancia.

Programa del Estado de la Nación. (2007). Décimo Tercer Informe del Estado de la Nación en Desarrollo humano sostenible. San José: Programa del Estado de la Nación.

Programa Estado de la Nación en Desarrollo Humano Sostenible. (2005). CONARE [Coord.]. Informe Estado de la Educación Costarricense (1). San José, Costa Rica: Programa del Estado de la Nación.

Stainback, Susan y Stainback, William. in inclusive classrooms: faciliting (1992). Curriculum considerations 
learning for all students. Baltimore: Paul Brookes.

Suaréz, G. (1998, marzo 23-25). La educación como elemento estratégico para el desarrollo de las naciones. Ponencia presentada en Simposio Costa Rica en el mundo: los próximos cincuenta años. [CD], (publicación digital, sin paginación). Centro de Investigación Docente y Educación, Universidad Nacional de Costa Rica.

Torres, J. (1996). Globalización e interdisciplinariedad: el currículo integrado ( ${ }^{a}$ ed.). Madrid: Morata. 